\title{
Enhanced production of antitumour naphthoquinones in transgenic hairy root lines of Lithospermum canescens
}

\author{
Katarzyna Sykłowska-Baranek • Agnieszka Pietrosiuk • \\ Anna Gawron - Anna Kawiak · Ewa Lojkowska • \\ Małgorzata Jeziorek · Ioanna Chinou
}

Received: 20 February 2011 / Accepted: 26 May 2011/Published online: 17 September 2011

(C) The Author(s) 2011. This article is published with open access at Springerlink.com

\begin{abstract}
The effects of medium exchange and methyl jasmonate addition on growth and production of shikonin and its derivatives acetylshikonin and isobutyrylshikonin in hairy root cultures of Lithospermum canescens were investigated. Responses varied depending on the transgenic line and stage of growth at which these lines were subjected to treatment. Shikonin itself was not detected, irrespective of the transgenic line and culture treatment used. A eightfold increase in acetylshikonin and isobutyrylshikonin accumulation was achieved when 32-day-old transgenic roots of Lc1D line were transferred from LS to M9 medium for the subsequent 3 weeks of culture. Methyl jasmonate exerted a detrimental effect on red naphthoquinones production. The extracts obtained from roots cultivated in M9 medium for 3 weeks were subjected to a cytotoxicity assay and displayed cytotoxic activity against
\end{abstract}

K. Sykłowska-Baranek $(\bowtie) \cdot$ A. Pietrosiuk · A. Gawron · M. Jeziorek

Department of Biology and Pharmaceutical Botany, Faculty of Pharmacy, Medical University of Warsaw, ul. Banacha 1, 02-097 Warsaw, Poland

e-mail: k.syklowska@yahoo.com

A. Kawiak · E. Łojkowska

Department of Biotechnology, Intercollegiate Faculty of Biotechnology, University of Gdańsk-Medical University of Gdańsk, ul. Kładki 24, 80-822 Gdańsk, Poland

\footnotetext{
A. Kawiak

Laboratory of Human Physiology, Faculty of Health Sciences with Subfaculty of Nursing, Medical University of Gdansk, Tuwima 15, 80-210 Gdansk, Poland

I. Chinou

Department of Pharmacognosy, School of Pharmacy, University of Athens, University Campus of Zografou, 15771 Zografou, Athens, Greece
}

human promyelocytic leukemia cells (HL-60) at the dose of $4 \mu \mathrm{g} \mathrm{ml}^{-1}$.

Keywords Cytotoxic activity - Hairy roots . Lithospermum canescens - Methyl jasmonate . Shikonin derivatives
Abbreviations
ACS Acetylshikonin
IBS Isobutyrylshikonin
DW Dry weight

\section{Introduction}

Transformation of plants with Agrobacterium rhizogenes is a commonly used method to enhance the production of secondary metabolites. Hundreds of species have been transformed with A. rhizogenes with the aim of achieving transformed root induction. The prospects for the production of medicinal compounds in transformed roots were previously reviewed (Georgiev et al. 2007; Guillon et al. 2006; Uozumi 2004). Several Boraginaceous species have been also transformed with $A$. rhizogenes for the production of shikonin derivatives. Lithospermum canescens hairy roots are a good example and the presence of some shikonin derivatives has been also reported in the transgenic roots of this species. The contents of acetylshikonin (ACS) and isobutyrylshikonin (IBS) in transformed roots of $L$. canescens made up ca. $10 \%$ of those observed in its natural roots (Pietrosiuk et al. 2006; Pietrosiuk and Wiedenfeld 2005). Recently, detailed chemical analysis of $L$. canescens transgenic roots has been performed and $\beta$-hydroxyvalerylalkannin and isobutylalkannin have been reported as novel metabolites within Lithospermum genus 
from both natural and transgenic roots (Graikou et al. 2011).

Biological studies of shikonin derivatives undertaken by many authors showed a broad spectrum of activity of these naphthoquinones, significant from the medicinal point of view, i.e. their antibacterial, antifungal, antiamoebic, antitumour, wound-healing, anti-inflammatory and immuno-stimulating properties. The effects produced by naphthoquinones have been attributed to the inhibition of topoisomerase I and II, protection from UV-radiation, stimulation of peroxidase, and inhibition of microsomal monooxygenase (Hisa et al. 1998; Papageorgiou et al. 2006a, b; Pietrosiuk et al. 2004a,b, 2003; Wagner et al. 1988).

Shikonin/alkanin was the first secondary metabolite produced on commercial scale using biotechnological methods (Papageorgiou et al. 1999). However, there have been still conducted investigations to reveal factors influencing its biosynthesis (Zou et al. 2011; Zhang et al. 2010; Liu et al. 2006) and to enhance shikonin/alkanin and their derivatives production (Zare et al. 2010).

In this study we investigated the time course study of root growth and shikonin derivatives accumulation in three transgenic root lines of $L$. canescens in order to identify the best candidate for the production of shikonin derivatives and to choose the optimal growth stage to achieve the highest red pigment productivity. Moreover, our study aimed at developing a satisfactory strategy leading to significant enhancement of red naphthoquinones production in hairy root cultures. We have also assessed the cytotoxic activity of the methanol extract from the examined transgenic root lines.

\section{Materials and methods}

Transgenic root lines and culture conditions

Hairy root cultures of L. canescens (Michx.) Lehm. were established using A. rhizogenes strains ATCC 15834, LBA 9402 and NCIB 8196 (Pietrosiuk et al. 2006). The bacteria were grown on YEB solid medium (Vervliet et al. 1975) $24 \mathrm{~h}$ at $24^{\circ} \mathrm{C}$, in the dark. Next, single colonies were inoculated into $50 \mathrm{ml}$ YEB liquid medium and cultured $72 \mathrm{~h}$ at $24^{\circ} \mathrm{C}$ in the dark, on a gyratory shaker (New Brunswick Scientific) at $120 \mathrm{rpm}$. The bacterial cultures were diluted (1:4) with YEB liquid medium before transformation. The leaves and stems of 6-week-old shoots were directly wounded with sterile needles containing bacterial suspension. The infected shoots were placed on hormonefree LS medium (Linsmaier and Skoog 1965) with the addition of sucrose $\left(30 \mathrm{~g} \mathrm{l}^{-1}\right)$ and solidified with $8 \mathrm{~g} \mathrm{l}^{-1}$ Phytagar (GIBCO). The incubation was performed at $25^{\circ} \mathrm{C}$ and under $40 \mu \mathrm{mol} / \mathrm{m}^{2} \mathrm{~s}^{-1}$ fluorescent lights for $18 \mathrm{~h}$ per day. The roots emerging from the infected sites were transferred to the liquid hormone-free LS medium supplemented with $0.05 \%$ Claforan (Roussel, France) after autoclaving and cultured individually at $25^{\circ} \mathrm{C}$ in the dark on a gyratory shaker (New Brunswick Scientific) at $120 \mathrm{rpm}$.

Three lines of transgenic roots: Lc1A, Lc1D and Lc1G resulted from transformation with strain ATCC 15834, were chosen for further investigations and stably maintained over successive subcultures. Every 6 weeks the roots were transferred to $50 \mathrm{ml}^{-1}$ of hormone-free liquid LS medium in $250 \mathrm{ml}$ Erlenmeyer flasks. The transgenic roots were incubated at $25^{\circ} \mathrm{C}$ in the dark on a INFORS AG TR 250 shaker at $105 \mathrm{rpm}$.

Determination of growth and shikonin derivatives accumulation

A time course study of hairy root growth and shikonin derivatives production were examined starting from day 4 up to day 42 of culture. The hairy roots were cultivated as described above. Every 3-4 days samples of each root line from two flasks were harvested. The biomass increase measured as dry weight, was investigated. The collected roots were gently pressed on filter paper to remove excess medium. Then they were lyophilized and their dry weight was recorded. The post-culture media were filtered and the pH of the media (pH-METR 5170 Elwro, Mera-tronik V 628 ), their conductivity (Elmetron) and sucrose levels were determined.

Treatment for enhancement of shikonin derivatives production

To improve shikonin derivatives accumulation, the transgenic roots were moved from LS medium to M9 medium and cultivated in $250 \mathrm{ml}$ Erlenmeyer flasks containing $50 \mathrm{ml}$ of liquid M9 medium (Fujita et al. 1981). The culture conditions were as described above. The 25-day-old or 32-day-old transgenic roots of each root line were moved to the M9 medium. After 1, 2 and 3 weeks of culture, roots from two flasks were taken.

To study the influence of methyl jasmonate (MJ) on shikonin derivatives accumulation the 25-day or 32-dayold transgenic roots were cultivated for 2 weeks in liquid M9 medium supplemented with $100 \mu \mathrm{M} \mathrm{MJ}$, added to the medium after autoclaving.

The collected roots were gently pressed on filter paper, lyophilized and their dry weight was determined. The postculture media were filtered and subjected to chemical analysis.

All experiments were conducted in three replications; in LS medium each repetition consisted of two flasks and in M9 medium each repetition consisted of three flasks. 
Chemical analysis

Shikonin derivatives were extracted from samples of three lines of $L$. canescens transgenic roots. For obtaining dye fractions, the powdered samples of lyophilized transgenic roots were sonicated with n-hexane. The extraction was done for $15 \mathrm{~min}$ at $40^{\circ} \mathrm{C}$. The media were also extracted with n-hexane $(2 \times 50 \mathrm{ml})$. The extracts were filtered through Whatman No. 2 paper and the solvent was evaporated from the extract solution under reduced pressure. Dry residue was dissolved in methanol and investigated in a DIONEX HPLC DAD system, equipped with an automated sample injector (ASI-100), and UVD 340S 1054 detector using the following conditions: gradient elution: acetonitrile $\quad(40-0 \mathrm{ml}) / 0.04 \mathrm{M}$ orthophosphoric acid (60-100 ml); flow rate $1.5 \mathrm{ml} \mathrm{min}^{-1}$; column: EC 250/4.6 Nucleosil 120-127 mm C18 (Macherey-Nagel), and monitoring eluent at 215, 278, 514, and $320 \mathrm{~nm}$. Shikonin (Wako) and its two derivatives ACS and IBS isolated previously from natural roots of $L$. canescens (Pietrosiuk and Wiedenfeld 2005) were used as the standards and analyzed under the same conditions. Peaks were assigned by spiking the samples with the standards and comparing of the retention times and UV spectra.

\section{Cytotoxicity assay}

The cytotoxic activity of extracts prepared from 32-day-old transgenic roots of $L$. canescens cultivated for 3 weeks in M9 medium was examined. The viability of the cell lines was determined using the MTT [(3-(4,5-dimethylthiazol-2yl)-2,5-diphenyltetrazolium bromide] assay.

The following cell lines were used: HL-60 (human promyelocytic leukemia), HeLa (human cervical carcinoma) and HaCaT (human keratinocyte line). The HL-60 and HeLa cell lines were obtained from the Department of Medical Biotechnology of the Intercollegiate Faculty of Biotechnology, University of Gdańsk-Medical University of Gdańsk. The HaCaT (human keratinocyte line) was obtained from the Department of Dermatology, Bispebjerg Hospital, Kopenhagen.

The HL-60 cell line was cultured in RPMI 1640 medium supplemented with $10 \%$ fetal bovine serum, $2 \mathrm{mM}$ glutamine, 100 units $/ \mathrm{ml}$ penicillin and $100 \mu \mathrm{g} \mathrm{ml}^{-1}$ streptomycin. The HeLa and the HaCaT cell lines were cultured in Dulbecco's modified Eagle's medium (DMEM) supplemented with $10 \%$ fetal bovine serum, $2 \mathrm{mM}$ glutamine, 100 units $\mathrm{ml}^{-1}$ penicillin and $100 \mu \mathrm{g} \mathrm{ml}^{-1}$ streptomycin The cultures were maintained in a humidified atmosphere with $5 \% \mathrm{CO}_{2}$ at $37^{\circ} \mathrm{C}$.

Cells were seeded in 96-well microtitre plates $\left(5 \times 10^{4}\right.$ cells/well) and treated for $24 \mathrm{~h}$ with the extracts in the concentration range $0-100 \mu \mathrm{g} \mathrm{ml}^{-1}$. MTT $\left(0.5 \mathrm{mg} \mathrm{ml}^{-1}\right)$ was added and the cells were incubated for $3 \mathrm{~h}$ at $37^{\circ} \mathrm{C}$ following their lysis with DMSO. Optical density of the formazan solution was measured at $550 \mathrm{~nm}$ with a plate reader (Victor, 1420 multilabel counter).

All chemicals were purchased from Sigma-Aldrich (St. Louis, MO, USA).

\section{Results and discussion}

Study of growth and shikonin derivatives production in LS medium

Three transgenic root lines of $L$. canescens were cultivated for 42 days in hormone-free LS liquid medium. The transgenic root growth rate was determined on the basis of their dry weight.

The growth pattern expressed by dry weight increases varied according to the investigated transgenic root line. The transgenic lines Lc1D and Lc1G started to grow without any lag phase typically observed in cell suspension culture (Fig. 1). The linear growth phase of the transgenic lines Lc1D and Lc1G started immediately and lasted for 32 and 40 days, respectively. During cultivation of transgenic root line Lc1D a short stationary phase between culture days 32 and 36 was noted and the fall in dry mass formation for the transgenic lines Lc1D and Lc1G began on the culture days 36 and 40, respectively. During the culture of Lc1A transgenic line the following growth pattern could be observed: 7-day lag phase, exponential growth phase form day 7 to day 25 , linear growth phase from day 25 to day 32 and 2-day long stationary growth phase from day 32 to day 36. Starting from day 36 a decline in growth was observed (Fig. 1).

The highest dry mass accumulation achieved by the transgenic root lines Lc1A, Lc1D and Lc1G was 10.4, 3.2 and $4.8 \mathrm{~g}^{-1}$ on days 32,36 and 40, respectively (Fig. 1).

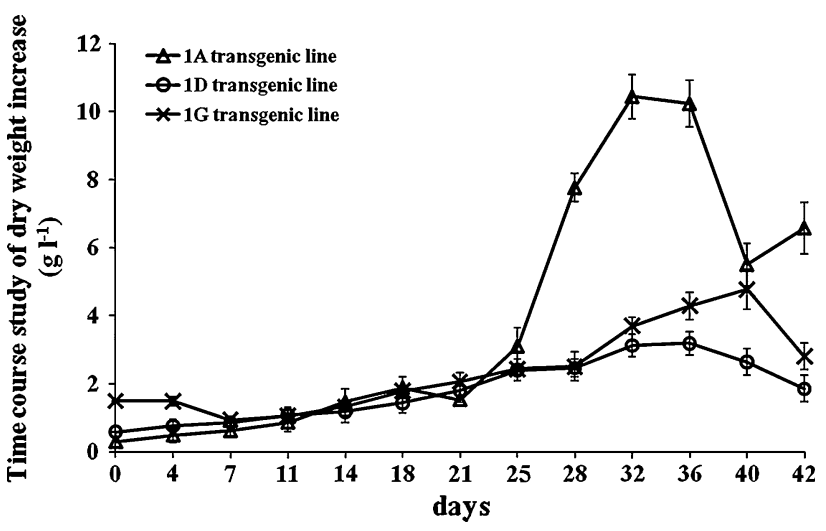

Fig. 1 Time course study of dry weight increase during cultivation of three $L$. canescens transgenic root lines in LS liquid medium 


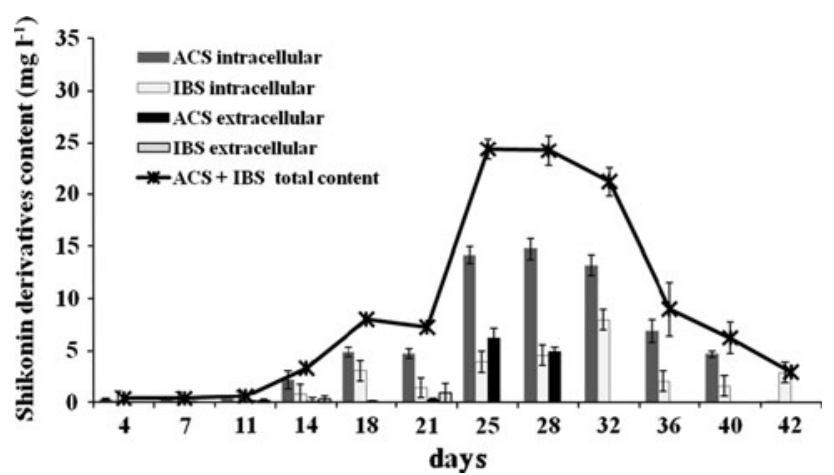

Fig. 2 Time course study of shikonin derivatives production during cultivation of transgenic root line Lc1A in LS liquid medium

However, the roots of line Lc1A differed morphologically from the roots of lines Lc1D and Lc1G and showed callus tissue formation.

The $\mathrm{pH}$ values changed slightly during the whole period of culture, irrespective of the root line. During culturing all transgenic root lines the decrease of sugar levels in the media was observed from the linear phase of their growth till the end of culture. The medium conductivity, as expected, negatively correlated with the root growth.

Two naphthoquinone, acetylshikonin (ACS) and isobutyrylshikonin (IBS), were extracted and quantified in the studied transgenic root lines. ACS was found to be the major shikonin derivative pigment in the transgenic lines whereas shikonin itself was not detected. ACS was also the main component of the red fraction isolated from natural roots of L. canescens (Pietrosiuk and Wiedenfeld 2005).

The process of ACS and IBS intracellular accumulation coincided with the dry mass increase in the cultures of Lc1D and Lc1G transgenic root lines (Figs. 1, 3, 4).

In the root cultures of line Lc1A, ACS and IBS concentrations started to increase from the beginning of culture and achieved the highest values in the linear growth phase (days 21-32) (Fig. 2).

The extracellular levels of ACS and IBS varied depending on the transgenic root line examined and the experimental period. In the post-culture media of the Lc1A transgenic line, the ACS content grew from day 7 to day 25 when the linear growth phase began and achieved its maximum value on day 25 (Fig. 2). IBS was present in the media from day 11 to day 21 day only with the highest yield on day 21 . From day 21 to the end of culture no IBS was found in the medium.

In the root line Lc1D, ACS was present in the medium till day 32 (the beginning of the stationary growth phase), but its highest yield was determined on day 21. In the medium, IBS was observed in very small quantities on days 28 and 32 only (Fig. 3).

During culturing of the Lc1G line roots, ACS could be determined in the medium throughout the culture duration

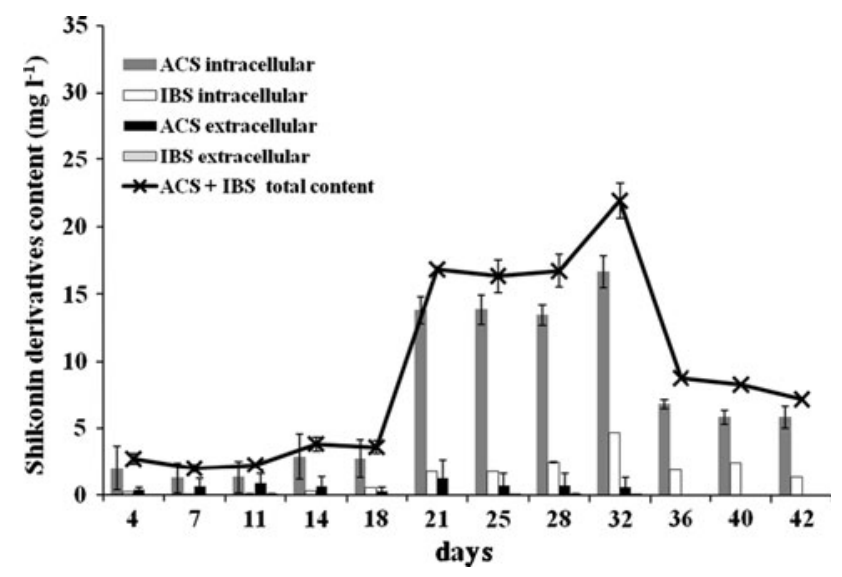

Fig. 3 Time course study of shikonin derivatives production during cultivation of transgenic root line Lc1D in LS liquid medium

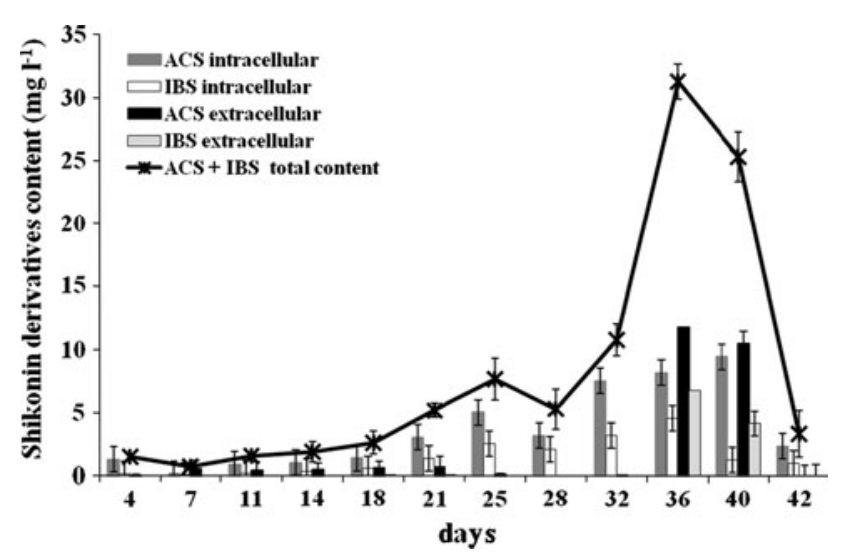

Fig. 4 Time course study of shikonin derivatives production during cultivation of transgenic root line Lc1G in LS liquid medium

except days 28 and 42 when no ACS was detected and reached its maximum content on day 36 . IBS could be found in the medium only on days 36 and 40 with the highest content on day 36 (Fig. 4).

The capacity to produce the two shikonin derivatives depended on the transgenic root line. Of all transgenic lines examined, the highest ACS and IBS total content (intracellular + extracellular) was found for the Lc1G transgenic line where it reached $31.27 \mathrm{mg}^{-1}$ on day 36 (Fig. 4), followed by the Lc1A transgenic line with $24.32 \mathrm{mg} \mathrm{l}^{-1}$ on day 25 (Fig. 2). During cultivating the transgenic line Lc1D, the highest total production of the two investigated compounds was observed on day 32 at $21.92 \mathrm{mg} \mathrm{l}^{-1}$ (Fig. 3). In our previous investigations the highest yield of ACS and IBS was detected in the Lc1D root line after 28 days of culture while there were no shikonin derivatives in Lc1G root extracts (Pietrosiuk et al. 2006). However, the instability of secondary metabolites production is often observed (Kim et al. 2004). Shimomura et al. (1991) investigating transgenic root cultures of Lithospermum 
erythrorhizon obtained the highest shikonin production after 4 weeks of culture (5.5 mg/flask). They also noticed that during the entire culture period ca. $25 \%$ of shikonin produced was released into the culture medium.

Study of M9 medium and methyl jasmonate effect on shikonin derivatives productivity

M9 medium, developed by Fujita et al. (1981) and successfully used for the enhancement of shikonin production in suspension culture of L. erythrorhizon, was employed to increase the production of shikonin derivatives in hairy root cultures of $L$. canescens. Considering the time course study of dry weight increase and of ACS and IBS accumulation it could be inferred that these two processes coincide and the highest shikonin derivative concentrations were determined during the linear growth phase between culture days 25 and 32 . To enhance ACS and IBS production the transgenic roots were transferred from LS to M9 medium on days 25 or 32 of culture and maintained in M9 medium for 3 weeks.

The transgenic roots continued growing during culturing in M9 medium (data not shown).

In M9 medium, the production of ACS and IBS increased significantly above their highest levels found in LS medium. The maximum concentrations of the two compounds were observed after 3 weeks of culture in M9 medium (Table 1). These two naphthoquinone pigments could be also found in the post-culture medium. However, the 25-day-old transgenic roots of the line Lc1A during cultivation in M9 did not excrete ACS and IBS into the post-culture medium.

Also no IBS was excreted into the medium in the cultures of 32-day-old Lc1A and Lc1G transgenic root lines growing in M9 medium while for the Lc1D transgenic line, IBS was detected in the medium only after 3 weeks of cultivating.
The levels of extracellular ACS in the post-culture media never exceeded $31 \%$ (Lc1G root line) and $21 \%$ (Lc1D root line) of its total content when the roots were transferred to M9 medium on day 25 of culture. When the medium transition took place on day 32 of culture transgenic roots of three examined lines excreted only trace amounts of ACS from 0.1 to $2.9 \%$ of its total content.

The effect of M9 medium on red naphthoquinones was the most pronounced in the culture of hairy roots of Lc1D line when the medium was changed on day 32 . The total content of ACS and IBS increased eightfold in comparison to the highest total yield of these compounds found when in LS medium was used. It was also the highest total ACS and IBS content detected during cultivation of the investigated transgenic root lines (Table 1).

Recently, Zhang et al. (2010) have investigated the expression of some genes involved in shikonin and its derivatives biosynthesis in cell suspension of $L$. erythrorhizon maintained under different culture conditions. They stated that the beneficial effect of medium transition from B5 to M9 on red pigments accumulation was connected with the rapid stimulation of genes crucial for the biosynthesis of shikonin its derivatives.

The M9 medium supplementation with methyl jasmonate resulted in a decrease in the culture growth. Also the ACS and IBS content was significantly lower $(P<0.05$, $t$ test) in all elicited 32-day-old transgenic root lines compared to those cultivated in M9 medium without the elicitor (Table 1). Methyl jasmonate added to M9 medium also caused a reduction in the growth of Onosma paniculatum cells in a suspension culture and simultaneously induced higher shikonin accumulation (Ding et al. 2004).

Methyl jasmonate also reduced the total ACS and IBS yield when added to the 25-day-old Lc1A and Lc1D root lines. However, cell suspension cultures of $L$. erythrorhizon in M9 medium with addition of methyl jasmonate $(50 \mu \mathrm{M})$

Table 1 Total acetylshikonin (ACS) and isobutyrylshikonin (IBS) production in three lines of L. canescens transgenic roots cultivated in M9 medium with or without $100 \mu \mathrm{M}$ methyl jasmonate

\begin{tabular}{|c|c|c|c|c|c|c|c|c|}
\hline \multicolumn{5}{|c|}{ M9 medium applied on day 25} & \multicolumn{4}{|c|}{ M9 medium applied on day 32} \\
\hline \multicolumn{5}{|c|}{$\begin{array}{l}\text { Total (intracellular }+ \text { extracellular) content } \\
\text { of ACS and IBS }\left(\mathrm{mg} \mathrm{l}^{-1}\right)\end{array}$} & \multicolumn{4}{|c|}{$\begin{array}{l}\text { Total (intracellular }+ \text { extracellular) content } \\
\text { of ACS and IBS }\left(\mathrm{mg} \mathrm{l}^{-1}\right)\end{array}$} \\
\hline \multirow{2}{*}{$\begin{array}{l}\text { Transgenic } \\
\text { root line }\end{array}$} & \multicolumn{3}{|l|}{ week } & \multirow{2}{*}{$\begin{array}{l}\text { Methyl } \\
\text { jasmonate } \\
\text { ( } 2 \text { weeks) }\end{array}$} & Week & & & \multirow{2}{*}{$\begin{array}{l}\text { Methyl } \\
\text { jasmonate } \\
\text { (2 weeks) }\end{array}$} \\
\hline & 1 & 2 & 3 & & 1 & 2 & 3 & \\
\hline Lc1A & $11.03 \pm 2.95$ & $33.75 \pm 3.41$ & $62.90 \pm 6.42$ & $47.42 \pm 7.20$ & $25.14 \pm 2.18$ & $34.45 \pm 2.96$ & $80.90 \pm 4.69$ & $8.59 \pm 0.69$ \\
\hline Lc1D & $107.51 \pm 11.40$ & $118.36 \pm 8.38$ & $100.14 \pm 9.14$ & $38.74 \pm 3.74$ & $48.12 \pm 6.76$ & $63.85 \pm 5.74$ & $182.01 \pm 9.87$ & $44.62 \pm 1.71$ \\
\hline $\mathrm{Lc} 1 \mathrm{G}$ & $29.64 \pm 6.18$ & $32.97 \pm 3.87$ & $43.20 \pm 4.81$ & $68.44 \pm 6.68$ & $41.26 \pm 3.24$ & $51.71 \pm 4.56$ & $123.38 \pm 9.02$ & $29.00 \pm 0.73$ \\
\hline
\end{tabular}

Data represent average values from 9 replicates \pm SD 
showed the content of ACS nearly two times higher than in the control (Gaisser and Heide 1996).

The results of this study are in opposite to other investigations where methyl jasmonane has been found a very potent agent to enhance secondary metabolites production in hairy root cultures (Frankfater et al. 2009; Kim et al. 2009; Kittipongpatana et al. 2002; Li et al. 2005; Zid and Orihara 2005).

The three investigated transgenic root lines differ in the excretion pattern of ACS and IBS in the presence of the elicitor. Line Lc1A roots did not release ACS or IBS into the medium irrespective whether they were 25-day-old or 32-day-old when placed into M9 medium containing methyl jasmonate. What is more, 32-day-old roots when cultivated in the medium with the elicitor did not produce IBS at all as ACS only could be detected.

Similarly, transgenic roots of line Lc1D did not excrete IBS into the medium as IBS could be found in the roots alone. ACS was found in both roots and the medium. However, this compound was mainly determined in the medium where it accounted for $57 \%$ and $63 \%$ of the total ACS content in culture of 25-day-old and 32-day-old roots, respectively.

Under the influence of the elicitor the transgenic roots of line Lc1G accumulated ACS and IBS in both the roots and the medium. The accumulation in the root tissue exceeded the levels in the medium. During culturing of 25-day-old roots 10 and $0.5 \%$ of the total ACS and IBS yield, respectively, could be detected in the medium. The 32-dayold roots excreted into the medium 3 and $16 \%$ of the total ACS and IBS production, respectively.

The extracts from 32-day-old transgenic roots cultivated in M9 medium for 3 weeks were submitted to a cytotoxicity assay against HL-60, HeLa and HaCaT cell lines.

It has been reported earlier that shikonin possesses tumour cell-killing activity against a variety of cell lines (Min et al. 2008; Wu et al. 2004; Zeng et al. 2009). Also ACS demonstrated antitumour effects in malignant cell lines (Xiong et al. 2009). ACS inhibited cell growth of human lung adenocarcinoma epithelial cells, human breast adenocarcinoma cell line and human hepatocellular carcinoma cell line in a dose dependent manner. The $\mathrm{IC}_{50}$ values were 5.6, 6.82 and 3,04 $\mu \mathrm{g} \mathrm{ml}^{-1}$, respectively. ACS also exerted an inhibitory effect on the growth of human gastric carcinoma cell line (Zeng et al. 2009).

Extracts used for the cytotoxicity assay in our study did not contain shikonin. The main component was ACS which accounted for at least $62 \%$ of the total pigment fraction. Our study revealed that extracts prepared from the transgenic roots of $L$. canescens displayed marked activity against HL-60 cells $\left(\mathrm{IC}_{50}=4 \pm 0.3 \mu \mathrm{g} \mathrm{ml}^{-1}\right.$ ) while the activity against HeLa and $\mathrm{HaCaT}$ cells was significantly lower, with the $\mathrm{IC}_{50}$ values of $20 \pm 1.2 \mu \mathrm{g} \mathrm{ml}^{-1}$ and $45 \pm 2.5 \mu \mathrm{g} \mathrm{ml}^{-1}$, respectively.

\section{Conclusions}

The results reported here show how the medium exchange can stimulate the shikonin derivatives accumulation in the transformed roots of $L$. canescens which seem to be a very promising alternative source for naphthoquinone pigment production. The three $L$. cansecens transgenic root lines produced the highest levels of shikonin derivatives when on day 32 they were transferred to M9 medium and cultured for 3 weeks. In these conditions, the total ACS and IBS content of the highest producing root line Lc1D (182.01 $\mathrm{mg} \mathrm{l}^{-1}$ ) was increased over eightfold. In our study we achieved a 4.7-fold increase in the total ACS and IBS yield compared to natural roots. However, the addition of methyl jasmonate caused both the reduction of biomass increase and shikonin derivatives yield.

Transgenic roots of $L$. canescens could be considered as a potent and abundant source of compounds demonstrating potential anticancer properties.

Acknowledgments This investigation was supported by a research grant No N N405 349933 from the Polish Ministry of Science and Higher Education.

Open Access This article is distributed under the terms of the Creative Commons Attribution Noncommercial License which permits any noncommercial use, distribution, and reproduction in any medium, provided the original author(s) and source are credited.

\section{References}

Ding J, Shi S, Jiang BH, Yang YH, Huang J, Shen HG, Xia K, Zhang J, Jiang X (2004) Effects of methyl jasmonate with indole-3acetic acid and 6-benzylamoniopurine on the secondary metabolism of cultured Ononsma paniculatum cells. In Vitro Cell Dev-P1 40:581-585

Frankfater CR, Dowd MK, Triplett BA (2009) Effect of elicitors on the production of gossypol and methylated gossypol in cotton hairy roots. Plant Cell Tissue Organ Cult 98:341-349

Fujita Y, Hara Y, Suga C, Morimoto T (1981) Production of shikonin derivatives by cell suspension cultures of Lithospermum erythrorhizon. II, A new medium for the production of shikonin derivatives. Plant Cell Rep 1:61-63

Gaisser S, Heide L (1996) Inhibition and regulation of shikonin biosynthesis in suspension cultures of Lithospermum. Phytochemistry 41:1065-1072

Georgiev MI, Pavlov AI, Bley T (2007) Hairy root type plant in vitro systems as sources of bioactive substances. Appl Microbiol Biotechnol 74:1175-1185

Graikou K, Baranek KS, Pietrosiuk A, Damianakos H, Jeziorek M, Chinou IB (2011) Chemical analysis of Lithospermum canescens (Michx.) Lehm. hairy roots through TLC, CC, GC-MS, ESI-MS and NMR methods. Acta Chromatogr 23:353-363

Guillon S, Trémouillaux-Guiller J, Pati PK, Rideau M, Gantet P (2006) Harnessing the potential of hairy roots: dawn of a new era. Trends Biotechnol 24:403-409

Hisa T, Kiura Y, Takada K, Suzuki F, Takigawa M (1998) Shikonin, an ingredient of Lithospermum erythrorhizon, inhibits angiogenesis in vivo and in vitro. Anticancer Res 18:783-790 
Kim BI, Gibon DM, Shuler ML (2004) Effect of subculture and elicitation and elicitation on instability of Taxol production in taxus sp. suspension cultures. Biotechnol Prog 20:1666-1673

Kim OT, Bang KH, Kim YC, Hyun DY, Kim MY, Cha SW (2009) Upregulation of ginsenoside and gene expression related to triterpene biosynthesis in ginseng hairy root cultures elicited by methyl jasmonate. Plant Cell Tissue Organ Cult 98:25-33

Kittipongpatana N, Davis DL, Porter JR (2002) Methyl jasmonate increases the production of valepotriates by transformed root cultures of Valerianella locusta. Plant Cell Tissue Organ Cult $71: 65-75$

Li W, Koike K, Asada Y, Yoshikawa T, Nikaido T (2005) Rosmarinic acid production by Coleus forskohlii hairy root cultures. Plant Cell Tissue Organ Cult 80:151-155

Linsmaier EF, Skoog F (1965) Organic growth factor requirements of tobacco tissue cultures. Physiol Plant 18:100-127

Liu Z, Qi JL, Chen L, Zhang MS, Wang XQ, Pang YJ, Yang YH (2006) Effect of light on gene expression and shikonin formation in cultured Onosma paniculatum cells. Plant Cell Tissue Organ Cult 84:39-46

Min R, Tong J, Wenjun Y, Wenhu D, Xiaojian Z, Jiacai H, Jian Z, Wantao C, Chenping Z (2008) Growth inhibition and induction of apoptosis in human oral squamous cell carcinoma Tca-8113 cell lines by shikonin was partly through the inactivation of NF$\mathrm{kB}$ pathway. Phytother Res 22:407-415

Papageorgiou VP, Assimpoulou AN, Couladouros EA, Hepworth D, Nicolaou KC (1999) The chemistry and biology of alkannin, shikonin, and related naphthazarin natural products. Angew Chem Int Edit 38:270-300

Papageorgiou VP, Assimopoulou AN, Samanidou VF, Papadoyannis IN (2006a) Recent advances in chemistry, biology and biotechnology of alkannins and shikonins. Curr Org Chem 10:21232142

Papageorgiou VP, Assimopoulou AN, Samanidou VF, Papadoyannis IN (2006b) Analytical methods for the determination of alkannins and shikonins. Curr Org Chem 10:583-622

Pietrosiuk A, Wiedenfeld $\mathrm{H}$ (2005) Shikonin derivatives from Lithospermum canescens. Pharm Biol 43:189-191

Pietrosiuk A, Kędzia B, Hołderna-Kędzia E, Wiedenfeld H, Malinowski M, Furmanowa M (2003) Antimicrobial activity of naphthoquinones from Lithospermum canescens (Michx.) Lehm. Herba Polonica 49:209-215

Pietrosiuk A, Furmanowa M, Skopińska-Różewska E, Sommer E, Skurzak H, Bany J (2004a) The effect of acetylshikonin isolated from Lithospermum canescens roots on tumor-induced cutaneous angiogenesis. Acta Poloniae Pharmaceutica-Drug Res 61:379-382

Pietrosiuk A, Skopińska-Różewska E, Furmanowa M, Wiedenfeld H, Sommer E, Sokolnicka I, Rogala E, Radomska-Leśniewska D,
Bany J, Malinowski M (2004b) Immunomodualtory effect of shikonin derivatives isolated from Lithospermum canescens on cellular and humoral immunity in Balb/c mice. Die Pharmazie 59:640-642

Pietrosiuk A, Sykłowska-Baranek K, Wiedenfeld H, Wolinowska R, Furmanowa M, Jaroszyk E (2006) The shikonin derivatives and pyrrolizidine alkaloids in hairy root cultures of Lithospermum canescens (Michx.) Lehm. Plant Cell Rep 25:1052-1058

Shimomura K, Sudo H, Saga H, Kamada H (1991) Shikonin production and secretion by hairy root cultures of Lithospermum erythrorhizon. Plant Cell Rep 10:282-285

Uozumi N (2004) Large-scale production of hairy root. Adv Biochem Eng Biotechnol 91:75-103

Vervliet G, Holsters M, Teuchy H, Van MM, Schell J (1975) Characterization of different plaque-forming and defective temperate phages in Agrobacterium. J Gen Virol 26:33-48

Wagner H, Kreher B, Jurcic K (1988) In vitro stimulation of human granulocytes and lymphocytes by pico- and femtogram quantities of cytostatic agents. Arzneim-Forsch/Drug Res 38:237-275

Wu Z, Wu LJ, Li LH, Tashiro SI, Onodera S, Ikejima T (2004) Shikonin regulates HeLa cell death via caspase-3 activation and blockage of DNA synthesis. J Asian Nat Prod Res 6:155-166

Xiong W, Luo G, Zhou L, Zeng Y, Yang W (2009) In vitro and in vivo antitumor effects of acetylshikonin isolated from Arnebia euchroma (Royle) Johnst. (Ruanzicao) cell suspension cultures. Chin Med 4:1-7

Zare K, Nazemiyeh H, Movafeghi A, Khosrowshahli M, MotallebiAzar A, Dadpour M, Omidi Y (2010) Bioprocess engineering of Echium italicum L.: induction of shikonin and alkannin derivatives by two-liquid-phase suspension cultures. Plant Cell Tissue Organ Cult 100:157-164

Zeng Y, Liu G, Zhou LM (2009) Inhibitory effect of acetylshikonin on human gastric carcinoma cell line SGC-7901 in vitro and in vivo. World J Gastroenterol 15:1816-1820

Zhang WJ, Su J, Tan MY, Liu GL, Pang YJ, Shen HG, Qi JL, Yang Y (2010) Expression analysis of shikonin-biosynthetic genes in response to M9 medium and light in Lithospermum erythrorhizon cell cultures. Plant Cell Tissue Organ Cult 101:135-1429

Zid SA, Orihara Y (2005) Polyacetylenes accumulation in Ambrosia maritima hairy root and cell cultures after elicitation with methyl jasmonate. Plant Cell Tissue Organ Cult 81:65-75

Zou A, Zhang W, Pan Q, Zhu S, Yin J, Tian R, Gu H, Wang X, Qi J, Yang Y (2011) Cloning, characterization, and expression of LeEIL-1, an Arabidopsis EIN3 homolog, in Lithospermum erythrorhizon. Plant Cell Tissue Organ Cult doi:10.1007/s11240010-9895-1 